\title{
Responsabilidad Social y Huella Ecológica: el caso de la Universidad Politécnica de San Luis Potosí
}

\author{
Juan Carlos Neri Guzmán* \\ Universidad Politécnica de San Luis Potosí \\ carlos.neri@upslp.edu.mx
}

José Luis Santos Morales

Acciona Osis

joseluis.santos.morales@acciona.com

\section{Mildreth Guadalupe Arriaga Alemán}

Instituto Tecnológico de San Luis Potosí

mildreth040793@hotmail.com

\author{
Revista Cultura Económica \\ Año XXXVIII • $\mathrm{N}^{\circ} 100$ \\ Diciembre 2020: 66-92
}

DOI: https://doi.org/10.46553/cecon.38.100.2020.p66-92

Resumen: Este artículo presenta el estudio de caso de la Universidad Politécnica de San Luis Potosí, como un ejemplo para dimensionar el impacto que llega a tener la oferta de servicios de educación superior sobre el medio ambiente. El cálculo se realiza a través de la herramienta Huella Ecológica, y permite conocer la cantidad de hectáreas de árboles que en promedio un integrante de la universidad debe plantar y mantener para compensar el desequilibrio que genera en el medio ambiente por la contaminación y consumo de recursos naturales, logrando así cubrir los requisitos para un desarrollo sustentable a través de la Responsabilidad Social Corporativa de la universidad.

Palabras clave: Responsabilidad Social Corporativa; Huella Ecológica; Universidad Politécnica de San Luis Potosí; Desarrollo sustentable

\section{Social Responsibility \& Ecological Footprint: the case of the Universidad Politécnica de San Luis Potosí}

Abstract: This article presents the case study of the Universidad Politécnica de San Luis Potosi as an example to size the impact that generates the offering of superior education services on the environment. The calculations are made through the tool "Huella Ecológica" (Ecological Footprint), this allows us to know the average number of tree hectares that one member of the university has to plant and take care of compensating the lack of equilibrium that generates on the environment due to contamination and consumption of natural resources, meeting this way the requirements for a sustainable development through Corporate Social Responsibility of the university.

Keywords: Corporate Social Responsibility; Ecological footprint; Universidad Politécnica de San Luis Potosí; Sustainable development

* Recibido: 25/08/2020 - Aprobado: 06/11/2020 


\section{Introducción}

Debido al incontrolable crecimiento de la población en el mundo, el elevado consumo de recursos naturales, así como las grandes cantidades de emisiones y residuos que produce la sociedad, se ha provocado un impacto ambiental negativo y devastador. El panorama es preocupante pues según el Fondo Mundial para la Naturaleza (WWF por sus siglas en inglés World Wildlife Fund, 2018) existe una disminución del 60\% en la población de especies vertebradas (de 1970 a 2014), una reducción de hasta un 83\% en la población de especies de agua dulce desde 1970, y el 75\% de la superficie de la Tierra ha sido sometida al impacto humano.

El daño al medio ambiente es cada vez más grave e irreversible, por esta razón los problemas ambientales ligados al desarrollo económico y social han adquirido mayor relevancia en la sociedad. Al día de hoy, algunas instituciones, empresas y gobiernos empiezan a actuar con soluciones que han venido de la mano con cambios tecnológicos, normas más estrictas, sanciones e impuestos a quien contamine; o se otorgan subsidios a quien elabore productos amigables con el ambiente, aun cuando son insuficientes los esfuerzos y están poco coordinados (Martínez Castillo, 2008).

Uno de los fenómenos que más ha incidido en el mal uso de los recursos tiene que ver con un inadecuado modelo de desarrollo y el crecimiento acelerado de las ciudades. Según el Reporte de Ciudades del Mundo (ONU-Habitat, 2016), actualmente el 54\% de la población mundial vive en ciudades, y para el 2050 esta proporción aumentará al 66\%, por lo que la presión sobre el medio ambiente y el uso de los recursos se intensificará. Asimismo, según la Friedrich-Ebert-Stiftung (FSE, 2019), la crisis social y ecológica que se vive en los países tiene su principal origen en los modos de vivir, producir y consumir, provocado por un pensamiento cortoplacista y reduccionista del desarrollo, que pone el crecimiento económico en el centro de la acción política y económica, y deja en un plano residual el bienestar de la población y el cuidado del medio ambiente, lo cual compromete las bases de vida de las futuras generaciones y de la preservación de la flora y la fauna como las conocemos.

Hemos sido testigos de cómo grandes empresas, sin un principio de responsabilidad social y poca ética en los negocios, han registrado abusos en el uso de los recursos (humanos, naturales y financieros), afectando negativamente a empleados, proveedores, clientes y el medio ambiente. Empresas que se localizan en zonas pobres con legislaciones poco exigentes, 
donde emplean mano de obra con menores niveles salariales, uso de empleo infantil, con escasa seguridad social, sin respetar medidas de contaminación, etc., las cuales obtienen grandes ganancias al vender sus productos con un alto valor de mercado, conocidas como plantas "sweatshops", lo que ha dado un mayor auge a la necesidad de incorporar prácticas de responsabilidad social (Ruiz Barrios, 2013).

Los retos que las ciudades están por enfrentar se relacionan con el uso de energías renovables, la reducción en el consumo de la energía, la modernización de la industria tradicional, la consolidación de Estados fuertes, mejora en la tributación sobre las utilidades y rentas del capital, la recuperación de los ecosistemas afectados, la mejora del transporte público, la dignificación de la vivienda, mejorar el tratamiento de los residuos, un nuevo ordenamiento territorial que priorice la protección de lo social, lo ambiental y lo público (FSE, 2019).

Para atender estos y otros problemas como la pobreza, las desigualdades y el cambio climático, la Organización de las Naciones Unidas promueve el cumplimiento de los Objetivos de Desarrollo Sostenible, entendiendo el desarrollo sostenible como aquel capaz de satisfacer las necesidades del presente sin comprometer la capacidad de las futuras generaciones para satisfacer sus propias necesidades (UN, 2015). No obstante, para lograr estas metas que buscan fomentar el progreso y la consolidación de ciudades prósperas en el largo plazo, se requiere el apoyo de los líderes de los diferentes países, así como de la participación de los diferentes agentes económicos como empresas, gobiernos y la propia sociedad civil organizada.

En términos prácticos, las acciones que los agentes económicos llevan a cabo para contribuir en el logro de una sociedad más justa y de proteger el ambiente se identifican con iniciativas de responsabilidad social, las cuales, hasta hoy, son voluntarias. Algunas de las vertientes de la responsabilidad social se identifican con mediciones de la huella ecológica, la huella hídrica, la huella del carbono, entre otras.

Esta investigación muestra los resultados del cálculo de la Huella Ecológica en la Universidad Politécnica de San Luis Potosí, como una medida del impacto que su infraestructura y población involucrada (estudiantes, profesores y administrativos) ejercen sobre el medio ambiente a través de la demanda de energía, papel, agua, movilidad y residuos- 
emisiones que se generan, y que requiere compensar para mantener el desarrollo sustentable de la ciudad.

La Universidad Politécnica de San Luis Potosí concentra una matrícula superior a 5,000 estudiantes y más de 300 empleados directos que diariamente realizan desplazamientos hacia la institución y demandan cantidades significativas de transporte, alimentos, agua, energía y otros servicios; así como también generan gran cantidad de residuos, lo cual tiene un impacto en la comunidad local y en el medio ambiente que los rodea.

Los resultados muestran que la Universidad Politécnica de San Luis Potosí genera un total de 2 mil 675 toneladas de dióxido de carbono, por lo que, para compensar esta contaminación, cada miembro debería sostener una superficie de 0.088 hectáreas de bosque (huella ecológica).

\section{Metodología}

Para la realización del cálculo de la huella ecológica se utiliza la metodología para universidades desarrollada por Noelia López Álvarez y aplicada en la Universidad de Santiago de Compostela (López, 2009). Esta metodología identifica los tipos de recursos y de residuos que hay que considerar en el cálculo de la huella ecológica en instituciones de educación superior.

El cálculo se realiza a partir de los datos documentados de los recursos naturales consumidos en la institución, como agua, superficie de construcción, papel y combustibles, como energía eléctrica, consumo de papel, y datos de salidas sobre producción de residuos urbanos y emisiones. Asimismo, de manera indirecta se recolecta información a partir de encuestas sobre consumo y disposición de papel y movilidad en estudiantes, personal docente y administrativo.

La información se maneja en términos de unidades emitidas de dióxido de carbono (CO2) con ayuda de factores de emisión ${ }^{2}$. El cálculo de la huella ecológica es el resultado de dividir el total de emisiones de dióxido de carbono emitidas en la universidad entre la capacidad de fijación de la masa forestal, a la cual se le suma el territorio de las instalaciones de la universidad. Finalmente, el resultado se convierte en términos de hectáreas de superficie de bosque necesarias para que puedan compensar las emisiones registradas ${ }^{3}$. 
La fórmula utilizada para calcular la huella ecológica anual por hectárea (Ha) para la universidad es la siguiente:

$$
\text { Huella }\left(\frac{h a}{\text { año }}\right)=\frac{\text { Emisiones }\left(\text { ton } \mathrm{CO}_{2}\right)}{\text { C.Fijación }\left(\frac{\text { tonCO }}{\frac{h a}{a \tilde{n} o}}\right)}+\text { SuperficieCampus }\left(\frac{h a}{a \tilde{n} o}\right)
$$

En este sentido, el objetivo de esta investigación es realizar el cálculo de la huella ecológica de la Universidad Politécnica de San Luis Potosí (UPSLP), como un compromiso de su responsabilidad social que permite identificar las fuentes que emiten dióxido de carbono (CO2) dentro de la universidad.

Este estudio propone como hipótesis que las emisiones de $\mathrm{CO} 2$ son mayores que la capacidad de absorción de áreas verdes dentro de la universidad, por lo que el indicador de la huella ecológica será un resultado negativo. Esto considerando la gran cantidad de alumnos que registra la universidad, no obstante, los estándares internacionales con los que opera.

Entre las preguntas que se busca contestar en esta investigación están ¿Qué es la responsabilidad social?, ¿Qué es la huella ecológica?, ¿Cuál es la huella ecológica de la Universidad Politécnica de San Luis Potosí?, ¿Qué fuentes tienen un mayor impacto negativo en el medio ambiente?

En este estudio se aborda, primeramente, el tema de la responsabilidad social como una estrategia que le permite a las instituciones llevar a cabo acciones para compensar el efecto negativo que sus actividades tienen sobre los empleados, la comunidad y el medio ambiente. Asimismo, se plantea el tema de la huella ecológica para verificar cómo esta herramienta nos permite cuantificar el impacto que tiene una institución sobre los recursos de la naturaleza, y se exponen algunos casos documentados. Por otra parte, también se presenta el modelo de gestión de la Universidad Politécnica de San Luis Potosí y sus resultados de la huella ecológica. Finalmente, se presentan las conclusiones del estudio. 


\section{Antecedentes teóricos y empíricos}

\section{La Responsabilidad Social}

La responsabilidad social representa un compromiso que una empresa debe asumir por el impacto social que generan las actividades que llevan a cabo, ya sean de tipo productiva, comercial o de servicio, lo que se refleja en acciones específicas para eliminar, minimizar o compensar en el caso de tratarse de un impacto negativo (EAE Business School, 2015).

La responsabilidad social está conformada por varios aspectos, uno de los más importantes es la responsabilidad social ambiental, que se identifica con la conservación y cuidado del medio ambiente a través de la implementación de programas de mitigación de emisiones de dióxido de carbono (Jiménez, 2014), midiendo los alcances que puede tener el cambio climático en su negocio, e incorporando mecanismos económicos para integrar el cambio climático a la toma de decisiones.

La responsabilidad social se trata de un compromiso por parte de los miembros de una sociedad, ya sea de manera individual o grupal, como una voluntad para la realización de actividades que tienen un impacto en el entorno. Más allá de buscar maximizar las ganancias, reducir los costos o mantener los precios, se busca llevar a cabo actividades complementarias o adicionales, que permitan reducir el impacto negativo que las actividades rutinarias pudieran tener tanto en el medio ambiente, como en el ámbito laboral e incluso, en la ética del comportamiento empresarial con los diversos grupos con los que se relacionan las empresas (Barroso Tanoira, 2008).

Para autores como Medina (2006), la responsabilidad social debe estar contemplada en la misión y visión de la empresa y se deben destinar recursos y esfuerzos necesarios para realizar un proyecto institucional que comprometa la participación de todos los involucrados para lograr la calidad en todas las acciones que realiza.

Por su parte, para el Centro Mexicano para la Filantropía (Cemefi), la responsabilidad social en una empresa significa buscar que sus operaciones sean sustentables e integrales en lo económico, lo social y lo ambiental, reconociendo los intereses de los distintos grupos con los que se relaciona y buscando la preservación del medio ambiente y la sustentabilidad de las generaciones futuras, independientemente de los productos o servicios que 
ofrece, del sector al que pertenece, de su tamaño o nacionalidad. Para ello, identifica seis dimensiones de la Responsabilidad Social Empresarial (Cajiga Calderón, 2020):

a. Dimensión económica interna, busca la generación y distribución del valor agregado entre colaboradores y accionistas, considerando no solo las condiciones de mercado sino también la equidad y la justicia. Se espera de la empresa que genere utilidades y se mantenga viva y pujante (sustentabilidad).

b. Dimensión económica externa, busca la generación y distribución de bienes y servicios útiles y rentables para la comunidad, además de su aportación a la causa pública mediante la contribución impositiva. Asimismo, la empresa debe participar activamente en la definición e implantación de los planes económicos de su región y país.

c. Dimensión social interna, implica la responsabilidad compartida y subsidiaria de inversionistas, directivos, colaboradores y proveedores para el cuidado y fomento de la calidad de vida en el trabajo y el desarrollo integral y pleno de todos ellos.

d. Dimensión sociocultural y política externa, conlleva a la realización de acciones y aportaciones propias y gremiales seleccionadas para contribuir con tiempo y recursos a la generación de condiciones que permitan y favorezcan la expansión del espíritu empresarial y el pleno desarrollo de las comunidades; $y$, por tanto, a un entorno de mercado favorable para el desarrollo de su negocio.

e. Dimensión ecológica interna, implica la responsabilidad total sobre las repercusiones ambientales de sus procesos, productos y subproductos; y, por lo tanto, la prevención -y en su caso remedio- de los daños que causen o pudieran causar.

f. Dimensión ecológica externa, conlleva a la realización de acciones específicas para contribuir a la preservación y mejora de la herencia ecológica común para el bien de la humanidad actual y futura.

En este sentido, la tendencia de la Responsabilidad Social Empresarial es una consideración que tienen los directivos de una empresa (o institución) para incluir en su filosofía de trabajo, el respeto al cuidado 
tanto de las condiciones internas como externas a la empresa en cuanto a los posibles efectos negativos que pudiera generar en lo social, económico y sobre el medio ambiente.

La responsabilidad social, también llega a convertirse en una ventaja competitiva para una empresa, cuando la adopta como estrategia para insertarse en un mercado internacional y que, al favorecer la creación de un entorno sano para operar competitivamente con un enfoque más humano y ecológico, logra mejorar el valor de la marca, la imagen, la reputación de la empresa y consolidarse en el mediano y largo plazo en los mercados locales y en las redes comerciales mundiales (Añez y Bonomie, 2010).

Por su parte, algunos especialistas hacen una diferencia entre Responsabilidad Social Empresarial y Responsabilidad Social Corporativa, distinguiendo a la primera por las acciones que una empresa lleva a cabo en favor de sus empleados, la sociedad y el medio ambiente, pero cuyo alcance se limita al ámbito y personal de la empresa. Mientras que, en la segunda, el compromiso se extiende a la cadena de valor de la actividad económica, hacia las comunidades locales y los problemas del medio ambiente, lo cual contempla ámbitos extraterritoriales y estándares internacionales (ver Tabla 1).

\section{Tabla 1. Responsabilidad Social Empresarial vs Responsabilidad Social Corporativa}

\begin{tabular}{|c|c|}
\hline sabilidad Social Emp & idad Social Co \\
\hline $\begin{array}{l}\text { Representa el compromiso de la } \\
\text { empresa por mejorar las condiciones } \\
\text { laborales de sus empleados, apoyo a las } \\
\text { causas humanitarias y cuidado al medio } \\
\text { ambiente, lo cual mejora su } \\
\text { productividad y le otorga una mayor } \\
\text { competitividad mejorando los siguientes } \\
\text { aspectos: } \\
\text { - Mejora de los productos y/o procesos } \\
\text { de producción, lo que resulta en una } \\
\text { mayor satisfacción y lealtad del cliente. } \\
\text { - Mayor motivación y fidelidad de los } \\
\text { trabajadores, lo cual aumenta su } \\
\text { creatividad e innovación. } \\
\text { - Mejor imagen pública debido a } \\
\text { premios y/o a un mayor conocimiento } \\
\text { de la empresa en la comunidad. } \\
\text { - Mejor posición en el mercado laboral y } \\
\text { mejor interrelación con otros socios }\end{array}$ & $\begin{array}{l}\text { Representa el compromiso de la empresa } \\
\text { por gestionar los impactos que su } \\
\text { actividad genera sobre sus clientes, } \\
\text { empleados, accionistas, comunidades } \\
\text { locales, medioambiente y sobre la } \\
\text { sociedad en general. En este sentido, el } \\
\text { beneficio es positivo para la empresa, } \\
\text { pero también para la sociedad, los } \\
\text { trabajadores y el medio ambiente. } \\
\text { La práctica de la Responsabilidad Social } \\
\text { Corporativa atiende a } 5 \text { principios: } \\
\text { - Cumplimiento de la legislación } \\
\text { nacional vigente y especialmente de } \\
\text { las normas internacionales. } \\
\text { - Carácter global, es decir afecta a todas } \\
\text { las áreas de negocio de la empresa y } \\
\text { las empresas en la cadena de valor, así } \\
\text { como a todas las áreas geográficas en } \\
\text { donde desarrolle su actividad. }\end{array}$ \\
\hline
\end{tabular}


empresariales y autoridades, mejor acceso a las ayudas públicas gracias a la mejor imagen de la empresa.

- Ahorro en costes e incremento de la rentabilidad debido a la mayor eficiencia en el uso de los recursos humanos y productivos.

- Incremento de la facturación/ventas como consecuencia de las prácticas implementadas.
- Compromisos éticos objetivos que se convierten en obligación para quien los contrae.

- Atención a los impactos que genera la actividad empresarial en el ámbito social, medioambiental y económico.

- Satisfacción e información de las expectativas y necesidades de los grupos de interés.

Los ámbitos que atiende la gestión se relacionan con derechos humanos, prácticas de trabajo y empleo, protección de la salud, cuestiones medioambientales, lucha contra el fraude y la corrupción, e intereses de los consumidores.

Fuente: Altagracia Martínez (2014) y ORSC (2014).

La manera en que una empresa o institución ejerce su responsabilidad social es, según Pérez Chavarría (2009), a través de alguna de las 7 modalidades que identifica en la Tabla 2:

Tabla 2. Modalidades de relación empresa y sociedad

\begin{tabular}{|c|l|}
\hline Modalidad & \multicolumn{1}{|c|}{ Descripción } \\
\hline $\begin{array}{c}\text { Acciones } \\
\text { complementarias }\end{array}$ & $\begin{array}{l}\text { Iniciativas que desarrolla la empresa y que están vinculadas } \\
\text { con sus requerimientos económico-comerciales, como: } \\
\text { programas de capacitación para empleados o reciclaje de } \\
\text { materiales. }\end{array}$ \\
\hline $\begin{array}{c}\text { Acciones } \\
\text { compensatorias }\end{array}$ & $\begin{array}{l}\text { Medidas adoptadas por la empresa para neutralizar los } \\
\text { efectos que produce su actividad en el medio ambiente } \\
\text { (sembrar árboles). }\end{array}$ \\
\hline Patrocinio & $\begin{array}{l}\text { Apoyo a un evento o espectáculos puntuales a cambio de un } \\
\text { espacio publicitario. }\end{array}$ \\
\hline Marketing con causa & $\begin{array}{l}\text { Estrategia que relaciona la promoción de un producto con } \\
\text { una causa de interés social. }\end{array}$ \\
\hline Mecenazgo & $\begin{array}{l}\text { Sostenimiento de actividades culturales abiertas a la } \\
\text { comunidad o apoyo a investigaciones científicas. }\end{array}$ \\
\hline Filantropía & Acciones benéficas (incluyen donaciones). \\
\hline Inversión social & $\begin{array}{l}\text { Interacción entre la empresa el medio. Supone el diseño y } \\
\text { puesta en marcha de algún proyecto conjunto (voluntariado } \\
\text { empresarial). }\end{array}$ \\
\hline
\end{tabular}

Fuente: Pérez Chavarría (2009).

Barrio Fraile (2016) presenta un variado catálogo de temáticas que se pueden realizar como actividades de responsabilidad social en función del tipo stakeholder ${ }^{4}$ de quien se trate (Tabla 3). Por ejemplo, para mejorar la relación con los clientes, una opción es realizar campañas de publicidad que 
no resulten engañosas sobre los bienes o servicios que se producen. Por su parte, para los empleados, una acción benéfica sería retribuir salarios justos, mientras que un beneficio para la sociedad será la reducción de residuos y emisiones contaminantes, entre muchas otras acciones.

\section{Tabla 3. Áreas de trabajo en materia de Responsabilidad Social Corporativa}

\begin{tabular}{|c|c|}
\hline & Stakeholder / Temáticas \\
\hline & $\begin{array}{l}\text { Clientes / consumidores } \\
\text { - Honestas relaciones comerciales [No publicidad engañosa e información exacta, } \\
\text { clara y comparable (etiquetado)]; -Innovación; -Calidad; - Fiabilidad; •Productos y } \\
\text { servicios seguros (cumplimiento de normas y salud y seguridad); -Precios } \\
\text { razonables; -Accesibilidad; •Privacidad; •Relación duradera (servicios de atención } \\
\text { al cliente, apoyo y resolución de quejas). }\end{array}$ \\
\hline & $\begin{array}{l}\text { Proveedores } \\
\text { - Medidas de apoyo y mejora; -Selección transparente, justa y responsable; •Precios, } \\
\text { cláusulas y expectativas equitativas. }\end{array}$ \\
\hline & $\begin{array}{l}\text { Empleados } \\
\text { - Cumplimiento de los derechos humanos (trabajo infantil, forzado y cárceles); -No } \\
\text { discriminación e igualdad de oportunidades por motivos de raza, color, sexo, } \\
\text { opinión política, edad, ascendencia nacional u origen social, etc. (Prácticas } \\
\text { responsables de contratación, Procesos de promoción interna, Misma retribución } \\
\text { entre hombres y mujeres); -Conciliación vida laboral-familiar; -Seguridad y salud } \\
\text { laboral; -Desarrollo humano y formación en el lugar de trabajo (Formación y } \\
\text { aprendizaje continuo y, Posibilidades de desarrollo profesional y promoción); } \\
\text {-Salarios justos; -Empleabilidad y perdurabilidad del puesto de trabajo; - -Libertad } \\
\text { de asociación y derecho de negociación colectiva; -Transparencia y comunicación; } \\
\text {-En caso de reestructuración, reducir los impactos negativos. }\end{array}$ \\
\hline & $\begin{array}{l}\text { Capital } \\
\text { - Creación de valor de forma sostenible; -Transparencia en la información; -Lealtad. }\end{array}$ \\
\hline & $\begin{array}{l}\text { Comunidad local } \\
\text { - Preservación del medio ambiente; -Compromiso con la sociedad local / } \\
\text { Contribución al desarrollo de la comunidad [Generación de riqueza e ingreso } \\
\text { (creación de empleo, salarios y prestaciones, ingresos fiscales, Colaboración con } \\
\text { proyectos comunitarios, Formación profesional, Contratación de personas } \\
\text { socialmente excluidas, Inversión social (donaciones), Educación y cultura]; } \\
\text {-Integración de la empresa en su entorno (Ser un buen vecino); -Desarrollo de } \\
\text { comunidades locales (Programas de desarrollo). }\end{array}$ \\
\hline & $\begin{array}{l}\text { Administración pública } \\
\text { - Colaboración de las empresas para cooperar en el desarrollo de una cultura de } \\
\text { Gestión ética y socialmente responsable; •Contribución a las finanzas públicas. }\end{array}$ \\
\hline & $\begin{array}{l}\text { Competencia } \\
\text { - Competencia leal; -Cooperación y alianzas (Sinergias). }\end{array}$ \\
\hline & $\begin{array}{l}\text { Sociedad en general } \\
\text { - Ámbito social [Lucha contra la corrupción, Control de los proveedores } \\
\text { (Cumplimiento de un comportamiento responsable y evaluación de la repercusión } \\
\text { social de los proveedores), Promoción de la RSC (colaboración con organizaciones } \\
\text { internacionales), Acción social, Mejorar su contribución a la sociedad, }\end{array}$ \\
\hline
\end{tabular}


Transparencia].

- Ámbito medio ambiental (Evaluación del impacto medio ambiental de los proveedores, producción (plantas producción y oficinas), logística (transporte productos) y consumidor, Sensibilización medioambiental a los proveedores, empleados y consumidores, Reducción del consumo de recursos naturales no renovables, Reducción de residuos y desechos, Reducción de emisiones contaminantes, Reducción del consumo de materias primas, Reducción de gastos energéticos, Promover una mayor responsabilidad ambiental.

Fuente: Barrio Fraile (2016)

Como se ha visto, la actividad humana, inconsciente y desorganizada, ha provocado cambios en el medio ambiente comprometiendo el desarrollo sustentable, debido a las grandes emisiones de gases efecto invernadero, las cuales, a su vez, han provocado un cambio climático que se ha reflejado en un aumento en la temperatura, sequías devastadoras en algunas regiones, el aumento del nivel del mar en ciudades costeras, entre otros muchos efectos negativos.

Entre los gases efecto invernadero se encuentran el dióxido de carbono (CO2), producto del uso de combustibles fósiles, el metano $\left(\mathrm{CH}_{4}\right)$, generado en las actividades agrícolas y los tiraderos, el óxido nitroso (N2O), generado por los procesos industriales y agrícolas, y los gases refrigerantes o clorofluorocarbonos, de más a menos abundantes y de menos a más peligrosos, respectivamente. Para Estados Unidos, la proporción de estos gases es de $85 \%, 8 \%, 5 \%$ y $2 \%$, en orden de presentación (Berners-Lee, 2011).

Existen diferentes metodologías para medir el cambio climático, entre las que se encuentran: la huella hídrica, la huella de carbono y la huella ecológica.

La huella hídrica, permite medir el impacto que genera el hombre sobre los recursos hídricos a través del volumen total de agua dulce que es utilizada para producir un bien o la prestación de un servicio (directa) a lo largo de toda su cadena de producción (indirecta), es decir, incorporando el uso y consumo de agua en cada etapa del proceso de producción, desde los insumos hasta el consumo final. Es decir, representa la cantidad de agua que nos cuesta fabricar un producto. La Huella Hídrica se mide en el volumen de agua consumida en aguas superficiales (calculada como agua azul), evaporada (calculada como agua verde) o contaminada (calculada como agua gris), ya sea en unidad de tiempo o en unidad de masa (Martínez Ruiz, 2013). 
La huella de carbono permite medir el impacto que una actividad o proceso de productos o servicios tiene sobre el cambio climático, a través de las emisiones (directas o indirectas) de gases de efecto invernadero generadas, en términos de $\mathrm{CO} 2$, en su ciclo de vida completo, desde la adquisición de insumos hasta su gestión como residuo (MMA, 2020). La huella de carbono se puede calcular para personas, organizaciones, productos, eventos o regiones territoriales, y puede realizarse ya sea por la Norma PAS 2050, la ISO/TS 14067 y/o bajo el GHG Protocol. Product Life Cycle Accounting and Reporting Standard.

Existen otras metodologías para medir el cambio climático, incluso la NASA ha propuesto una manera alternativa para medir el calentamiento de la tierra, basada en el uso de la información de los satélites, midiendo el equilibrio entre la radiación solar que ingresa a la atmósfera de la Tierra y la cantidad absorbida, reflejada y emitida, donde, si la Tierra absorbe más calor del que emite, se calienta (NASA, 2020).

Asimismo, existen acciones a nivel global como el Pacto Mundial, el Pacto de Paris y el Pacto Global de Alcaldes por el Clima y la Energía para atender la mitigación del cambio climático, la adaptación a los efectos adversos del cambio y el acceso universal a energía segura, limpia y asequible. En México tenemos el caso de la Alianza por la Responsabilidad Social Empresarial (AliaRSE).

\section{La huella ecológica}

Los pioneros del concepto de huella ecológica en los años 90's fueron Wackernagel y Rees (1996), quienes mencionan que la huella ecológica compara la demanda humana sobre los recursos de la naturaleza contra la capacidad de la biosfera para regenerar esos recursos y proporcionar bienes y servicios ecológicos. También se puede entender como el "número máximo de individuos que es capaz de soportar de forma indefinida un hábitat sin alterar su productividad" (EOI, 2012: capítulo 3).

La idea de la huella ecológica es estimar la cantidad de recursos naturales que un individuo debería disponer para compensar el gasto que registra en estos recursos y la contaminación que genera, y no comprometer su existencia o la de sus descendientes en el futuro. En 1961, Arvill estimó que un individuo necesitaba 1 hectárea para lograr su mantenimiento. Por su parte, en 1993, P. y A. Ehrlich estimaron que una ciudad de un millón de habitantes, necesitaba 1,000 km2 para cubrir sus necesidades, lo que 
equivale a que cada individuo requiere de 1,000 $\mathrm{m} 2$ de superficie para asegurar su existencia en equilibrio con la naturaleza (VCS, 2014).

En este sentido, la huella ecológica es una métrica que compara la demanda de recursos de individuos, gobiernos y empresas contra lo que la Tierra puede renovar. Por tanto, un valor mayor de huella ecológica, significa un mayor consumo de recursos que registra un habitante, lo cual es un indicador negativo, pues refleja que el hombre consume y utiliza más cantidad de materiales y tierra que la que hay en su territorio, lo que representa un déficit ecológico.

Existen datos de gran interés al respecto, por ejemplo, un habitante promedio en el mundo tiene una huella de carbono anual de 7 toneladas, mientras un norteamericano tiene una de 28 toneladas, un británico de 15 toneladas y un chino de 3 toneladas. Asimismo, por cada tonelada de gases efecto invernadero que genera un habitante en México, un español genera 2 toneladas y un árabe genera 3 toneladas. Desde otra perspectiva podemos ver que una bolsa de papel procesada genera 4.4 veces más dióxido de carbono que una bolsa reciclada, o una unidad de calor generada por un boiler viejo genera 8 veces más dióxido de carbono que una generada por energía solar (Berners-Lee, 2011).

The Global Footprint Network realiza un cálculo tanto de la huella ecológica como del déficit de reservas ecológicas que registran los países tomando en cuenta diferentes variables como la superficie edificada, volumen de emisiones de carbono, tierras dedicadas a cultivos, extensión de la zona de pesca, productos forestales, y tierras de pastoreo. Para el año 2016, la huella ecológica per cápita por países con valores más altos, correspondieron a Qatar, Luxemburgo, Emiratos Árabes Unidos, Bahrein, Kuwait, Trinidad y Tobago, Estados Unidos, Canadá y Micronesia, con montos mayores a 7,600 hectáreas por persona. México ocupó el lugar número 91 de 189 países con 2,603 hectáreas por persona (Figura 1). 
Figura 1. Huella Ecológica per cápita (en hectáreas globales, 2016)

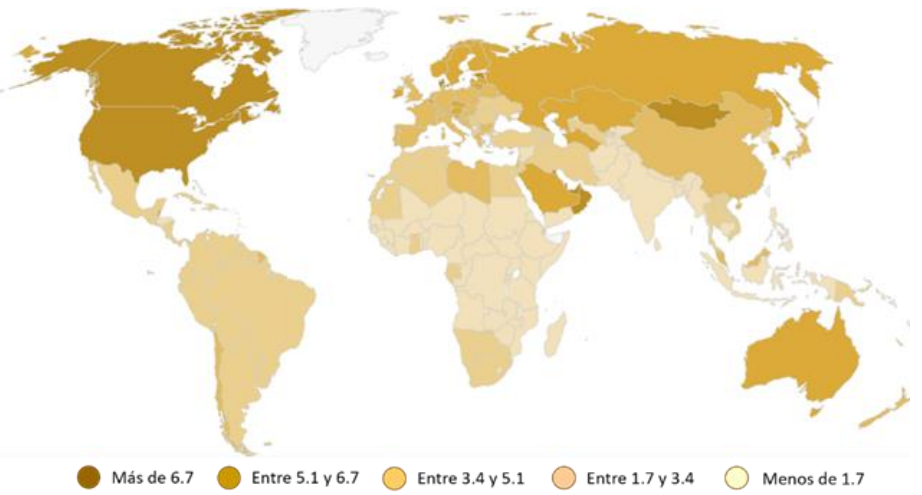

Nota. Considerando que la biocapacidad por persona es de 1.7 hectáreas, un rango de 6.8 hectáreas, por ejemplo, significa que un ciudadano está exigiendo cuatro veces los recursos y desechos que el planeta puede regenerar y absorber en la atmósfera.

Fuente: The Global Footprint. https://www.footprintnetwork.org/

Asimismo, la responsabilidad social también se identifica con sectores específicos, como en el caso de las instituciones de educación superior, en las cuales se llevan a cabo actividades relacionadas con la atención a las necesidades de la sociedad, lo que algunos llaman "Responsabilidad Social Académica” (Anand, 2019).

En este caso, la responsabilidad social universitaria, se refleja en la habilidad y efectividad de la Universidad para responder a las necesidades de la sociedad donde está inmersa, a través de sus funciones sustantivas: docencia, investigación, generación de conocimiento, extensión y gestión interna, con un compromiso de transferir en bien de la comunidad el saberhacer-cambiar los problemas o atender las necesidades, lo que le demanda una mayor autonomía a la universidad, así como la disposición de infraestructura de investigación y desarrollo (Almeida y Arrechavaleta Guarton, 2018). En este caso, Etzkowitz et al. (2008) identifica que las universidades requieren evolucionar para cumplir con el compromiso (y necesidad) que tienen para coadyuvar en la resolución de los problemas regionales, sectoriales, prioritarios o estratégicos de la sociedad, como consolidar la industria, superar el estancamiento, frenar la salida de capitales, evitar la concentración de la riqueza o disminuir el aumento de la pobreza, entre otras prioridades. 
Son diversas las maneras en que una universidad muestra su responsabilidad social, por ejemplo, el Tecnológico de Estudios Superiores de Coacalco contempla en su Plan de Responsabilidad Social Universitaria (León Borges et al., 2019), acciones como donaciones, clasificación de la basura, proyectos de incorporación de personas de la tercera edad, talleres para niños, ahorro de energía, programas de reforestación y cuidado con el agua, incorporando la participación de los estudiantes, personal docente y administrativo (García Molina, 2019). Pero, es importante adoptar medidas de responsabilidad social orientadas a mitigar los factores que más inciden negativamente en el medio ambiente, de aquí la relevancia de un estudio ad hoc como este.

Asimismo, existen diferentes estudios que las universidades realizan para medir su huella ecológica, como un indicador del grado de responsabilidad que estas instituciones mantienen ante el cuidado del medio ambiente.

\section{Huella Ecológica en las Universidades del mundo}

En los siguientes casos de Huella Ecológica (HE) se muestran los resultados obtenidos en diferentes universidades del mundo, en cada uno se detalla el nombre de la universidad, su localización, la metodología, variables e indicadores utilizados, y los resultados.

Tabla 4. Huella ecológica en la Universidad de Santiago de Compostela

\begin{tabular}{|c|l|}
\hline Caso & \multicolumn{1}{|c|}{ Universidad de Santiago de Compostela } \\
\hline Localización & España \\
\hline $\begin{array}{c}\text { Metodología } \\
\text { utilizada }\end{array}$ & $\begin{array}{l}\text { López Álvarez, N. Metodología para el Cálculo de la huella } \\
\text { ecológica en universidades. Congreso Nacional del Medio } \\
\text { Ambiente. Universidad de Santiago de Compostela. Oficina de } \\
\text { Desarrollo Sostenible, 20o9. }\end{array}$ \\
\hline $\begin{array}{c}\text { Variables } \\
\text { utilizadas }\end{array}$ & $\begin{array}{l}\text { Consumo de agua, construcción de edificios, energía eléctrica, } \\
\text { energía calorífica, movilidad, consumo de papel, generación de } \\
\text { residuos, generación de residuos peligrosos. }\end{array}$ \\
\hline $\begin{array}{c}\text { Indicadores } \\
\text { utilizados para el } \\
\text { cálculo }\end{array}$ & $\begin{array}{l}\text { Emisiones } \mathrm{CO}_{2} \text { (t/año), capacidad de fijación }\left(\mathrm{t} \mathrm{CO}_{2} / \mathrm{Ha} / \text { año) y }\right. \\
\text { área de absorción requerida (ha) }\end{array}$ \\
\hline Resultados & $\begin{array}{l}\text { La Huella Ecológica en la universidad en el año 2007 fue de } \\
5,217 \text { ha de bosque. }\end{array}$ \\
\hline
\end{tabular}

Fuente: López Álvarez (2009). 


\section{Tabla 5. Huella ecológica en la Universidad Central "Marta Abreu" de Las Villas}

\begin{tabular}{|c|l|}
\hline Caso & \multicolumn{1}{|c|}{ Universidad Central "Marta Abreu" de Las Villas } \\
\hline Localización & Santa Clara, Cuba \\
\hline $\begin{array}{c}\text { Metodología } \\
\text { utilizada }\end{array}$ & $\begin{array}{l}\text { López Álvarez, N. Metodología para el Cálculo de la huella } \\
\text { ecológica en universidades. Congreso Nacional del Medio } \\
\text { Ambiente. Universidad de Santiago de Compostela. Oficina de } \\
\text { Desarrollo Sostenible, 2009. }\end{array}$ \\
\hline $\begin{array}{c}\text { Variables } \\
\text { utilizadas }\end{array}$ & $\begin{array}{l}\text { Consumo de agua, construcción de edificios, energía eléctrica, } \\
\text { movilidad, generación de vapor, consumo de papel, consumo de } \\
\text { alimentos, generación de residuos, área ocupada directamente } \\
\text { por edificios. }\end{array}$ \\
\hline $\begin{array}{c}\text { Indicadores } \\
\text { utilizados para el } \\
\text { cálculo }\end{array}$ & $\begin{array}{l}\text { Emisiones } \mathrm{CO}_{2} \text { (t/año), capacidad de fijación }\left(\mathrm{C} \mathrm{CO}_{2} / \mathrm{Ha} / \text { año) y }\right. \\
\text { área de absorción requerida (ha) }\end{array}$ \\
\hline Resultados & $\begin{array}{l}\text { La Huella Ecológica en la Universidad Central "Marta Abreu" de } \\
\text { Las Villas en el año 2008 fue de 1,754.63 ha de bosque, } \\
\text { equivalente a o,2152 ha por persona. }\end{array}$ \\
\hline
\end{tabular}

Fuente: Leiva Mas, Rodríguez Rico y Martínez Nodal (2012).

\section{Tabla 6. Huella ecológica en la Universidad Nacional Autónoma de México}

\begin{tabular}{|c|c|}
\hline Caso & $\begin{array}{c}\text { Campus Zaragoza de la Universidad Nacional Autónoma de } \\
\text { México }\end{array}$ \\
\hline Localización & México \\
\hline $\begin{array}{l}\text { Metodología } \\
\text { utilizada }\end{array}$ & $\begin{array}{l}\text { - Vega García, S. Modelo de cuestionario para el cálculo de la } \\
\text { Huella Ecológica y su aplicación a estudiantes de la } \\
\text { Carrera de Biología. Tesis de Licenciatura en Biología. } \\
\text { (Facultad de Estudios Superiores Zaragoza, Universidad } \\
\text { Nacional Autónoma de México, México, D.F., pp.98, 2013). } \\
\text { - González-Álvarez, J. Colina-Vuelta, A. \& García-de la Fuente, } \\
\text { L. Análisis de la Huella Ecológica en el Principado de Asturias } \\
\text { (2010). Recuperado el } 3 \text { de marzo de } 2012 .\end{array}$ \\
\hline $\begin{array}{l}\text { Variables } \\
\text { utilizadas }\end{array}$ & $\begin{array}{l}\text { Basado en los consumos más frecuentes del mexicano promedio } \\
\text { en cuanto a alimentación, uso de energía eléctrica y gas } \\
\text { doméstico, medios de transporte, consumo de papel y superficie } \\
\text { de infraestructura urbana utilizada. }\end{array}$ \\
\hline $\begin{array}{c}\text { Indicadores } \\
\text { utilizados para el } \\
\text { cálculo }\end{array}$ & $\begin{array}{l}\text { Emisiones } \mathrm{CO}_{2} \text { (t/año), capacidad de fijación }\left(\mathrm{t} \mathrm{CO}_{2} / \mathrm{Ha} / \text { año) y }\right. \\
\text { área de absorción requerida (ha) }\end{array}$ \\
\hline Resultados & $\begin{array}{l}\text { Obteniendo una Huella Ecológica de } 1.48 \text { hag, con resultado de } \\
\text { mayor impacto ambiental individual al rubro de alimentos (o.42 } \\
\text { hag) por el consumo de cárnicos, seguido del uso de energía } \\
\text { (o.35 hag), principalmente por utilizar gas LP en la ducha } \\
\text { cotidiana. Esta HE está dentro de los límites de sustentabilidad } \\
\text { global (< } 1.61 \text { hag). }\end{array}$ \\
\hline
\end{tabular}

Fuente: Ibarra Cisneros y Monroy Ata (2014). 


\section{Análisis de resultados}

La Universidad Politécnica de San Luis Potosí (UPSLP) fue creada el 27 de junio de 2001 como un organismo descentralizado del Gobierno del Estado con apoyo del Gobierno Federal, y dio inicio al Subsistema de Universidades Politécnicas, actualmente formado por más de 60 universidades, todas integradas bajo la Coordinación General de Universidades Politécnicas y Tecnológicas (CGUTyP, 2020).

La oferta educativa de la UPSLP contempla los programas de Ingeniería en Sistemas y Tecnologías Industriales (ISTI), Ingeniería en Tecnologías de la Información (ITI), Ingeniería en Telemática (ITEM) e Ingeniería en Tecnologías de Manufactura (ITMA); así como programas de Licenciatura en Administración y Gestión (LAG) y Licenciatura en Mercadotecnia Internacional (LMI).

La UPSLP inició operaciones de instalaciones provisionales en inmuebles ubicados en el centro histórico de la capital, y la construcción de sus instalaciones se realizó bajo el Proyecto de Prestación de Servicios (PPS) 5 , mediante licitación pública internacional con una capacidad para 5,000 estudiantes.

En 2007 se asignó el contrato para el diseño, construcción, suministro de equipos, financiamiento, mantenimiento y operación de la Universidad, por un período de 20 años, a la empresa española Acciona. De alcance global, la empresa tiene presencia en los cinco continentes y opera con estándares internacionales de responsabilidad social y prácticas rigurosas de gestión ambiental ${ }^{6}$, con un modelo de negocio construido en torno a la sostenibilidad y en atención a los mayores desafíos globales, como el calentamiento global, la superpoblación y la escasez hídrica, los cuales atiende con soluciones en energía renovable, infraestructuras resilientes, gestión y tratamiento de agua, y atención a servicios. En la Universidad Politécnica de San Luis Potosí, Acciona gestiona 20 diferentes servicios (Tabla 7).

Tabla 7. Servicios en contrato de Acciona en la Universidad Politécnica de San Luis Potosí

\begin{tabular}{|l|l|l|l|}
\hline \multicolumn{4}{|c|}{ Servicios concesionados (no educativos) } \\
\hline $\begin{array}{l}\text { - Administración } \\
\text { de Servicios }\end{array}$ & $\begin{array}{l}\text { Administración } \\
\text { de Servicios } \\
\text { Públicos y }\end{array}$ & $\begin{array}{l}\text { - Mantenimiento } \\
\text { de Instalaciones }\end{array}$ & $\begin{array}{l}\text { Jardinería y } \\
\text { Mantenimiento de } \\
\text { Áreas Exteriores }\end{array}$ \\
\hline
\end{tabular}




\begin{tabular}{|l|l|l|l|}
\hline Operacionales & Energía & y Equipamiento & - Telecomunicaciones \\
- Vigilancia & - Limpieza & - Impuestos & - Gestión de \\
- Recepción & - Retiro de Basura & Locales & Emergencias \\
- Impresión, & - Control de & - Cafetería & - Continuidad de \\
Copiado y & Plagas & - Servicio de Café & Servicios \\
Papelería & - Centro de & - Venta de & Educativos \\
- Mensajeros y & Atención al & Alimentos & - Intendencia \\
Correos & Usuario & Ligeros y & \\
& & Bebidas & \\
& & & \\
\hline
\end{tabular}

Fuente: Suárez, S. (2009) Acciona concesiones: Proyecto de provisión de servicios (PPS) Universidad Politécnica de San Luis Potosí.

La construcción de la Universidad Politécnica de San Luis Potosí se realizó en cuatro etapas ${ }^{7}$ y fue ejercida con una inversión estimada de 3,306,000,00o MXN, siendo inaugurada el día 19 de septiembre del 2008, por el entonces Presidente de México, Felipe Calderón Hinojosa, y el ex Gobernador del Estado, Marcelo de los Santos. Las operaciones iniciaron el 21 de octubre de 2008 (Suárez, 2009). Actualmente, la UPSLP cuenta con una superficie de 15.6 hectáreas donde se localizan 15 edificios para brindar servicios a maestros, administrativos y alumnos, con 60,000 m2 de construcción, 6.45 hectáreas de áreas de reserva, una capacidad de 1,000 espacios de estacionamiento, incluyendo 30 para discapacitados (Figura 2).

\section{Figura 2. Distribución de edificios en la Universidad Politécnica de San Luis Potosí}

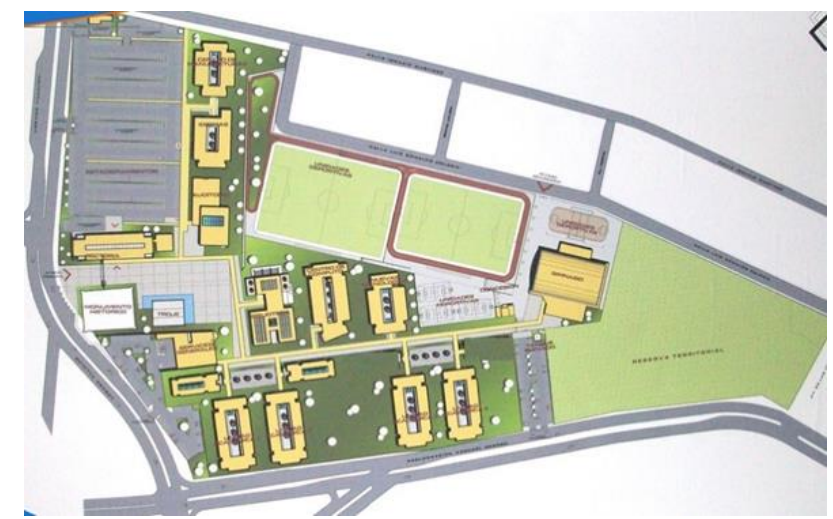

Fuente: Universidad Politécnica de San Luis Potosí. Dirección de Recursos Materiales (2020)

Las características de las instalaciones de la Universidad Politécnica de San Luis Potosí y su capacidad de servicios se detallan en la Tabla 8. 


\section{Tabla 8. Características de la infraestructura de la Universidad Politécnica de San Luis Potosí}

\begin{tabular}{|c|c|c|}
\hline Edificio & Descripción & $\begin{array}{l}\text { Superficie/ } \\
\text { Capacidad }\end{array}$ \\
\hline Rectoría & $\begin{array}{l}\text { Edificio de } 2 \text { plantas con } 54 \text { cubículos, } 27 \\
\text { estaciones de trabajo. Planta baja: atención a los } \\
\text { alumnos. Planta alta: rector y directores de la } \\
\text { universidad. }\end{array}$ & $\begin{array}{l}3,127.84 \mathrm{~m}^{2} \\
\text { Capacidad } \\
\text { para } 259 \\
\text { usuarios. }\end{array}$ \\
\hline $\begin{array}{c}\text { Biblioteca y } \\
\text { Cafetería }\end{array}$ & $\begin{array}{l}\text { Edificio de } 2 \text { plantas con cafetería en planta baja } \\
\text { para } 200 \text { comensales. Planta baja con salas de } \\
\text { lectura individual, estudio grupal, estaciones de } \\
\text { consulta en línea, oficinas y Acervo } 1 \text {. Planta alta } \\
\text { con } 3 \text { salas de lectura para 500 usuarios, } \\
\text { cableado estructurado y AC central, Acervo } 2 \text {. }\end{array}$ & $\begin{array}{l}3,451.48 \mathrm{~m}^{2} \\
\text { Capacidad } \\
\text { del edificio } \\
\text { biblioteca } \\
\text { de } 640 \\
\text { usuarios. }\end{array}$ \\
\hline $\begin{array}{l}\text { Centro de } \\
\text { Nuevas } \\
\text { Tecnologías }\end{array}$ & Planta baja con 9 laboratorios y planta alta con 7 . & $\begin{array}{l}2,666.67 \mathrm{~m}^{2} \\
\text { Capacidad } \\
\text { para } 550 \\
\text { alumnos. }\end{array}$ \\
\hline $\begin{array}{l}\text { Centro de } \\
\text { Cómputo }\end{array}$ & $\begin{array}{l}\text { Planta baja con } 10 \text { laboratorios y } 3 \text { cubículos de } \\
\text { trabajo. Planta alta con } 9 \text { laboratorios, } 5 \\
\text { cubículos de trabajo y site central. }\end{array}$ & $\begin{array}{l}3,650.23 \mathrm{~m}^{2} \\
\text { Capacidad } \\
645 \\
\text { alumnos y } \\
105 \\
\text { usuarios. }\end{array}$ \\
\hline $\begin{array}{l}\text { Unidad } \\
\text { Académica de } \\
\text { Maestros }\end{array}$ & $\begin{array}{l}4 \text { Unidades académicas con área de trabajo } \\
\text { electrónica. Capacidad } 645 \text { alumnos y } 105 \\
\text { usuarios y una sala de dirección. Capacidad para } \\
78 \text { profesores, } 43 \text { cubículos, sala de maestros y } \\
\text { de juntas, cada una. }\end{array}$ & $\begin{array}{l}1,240.05 \mathrm{~m}^{2} \\
\text { por Unidad } \\
\text { Capacidad } \\
645 \\
\text { alumnos y } \\
105 \\
\text { usuarios. }\end{array}$ \\
\hline $\begin{array}{l}\text { Unidad } \\
\text { Académica de } \\
\text { Estudiantes }\end{array}$ & $\begin{array}{l}4 \text { Unidades de estudiantes, con recepción y } \\
\text { jardín interior, con } 24 \text { aulas y } 2 \text { laboratorios cada } \\
\text { una. Cada unidad se divide en } 2 \text { plantas. }\end{array}$ & $\begin{array}{l}3,001.98 \mathrm{~m}^{2} \\
\text { cada una. } \\
\text { Capacidad } \\
\text { para } 800 \\
\text { alumnos. }\end{array}$ \\
\hline $\begin{array}{l}\text { Centro de } \\
\text { Manufactura } \\
\text { Avanzada }\end{array}$ & $\begin{array}{l}10 \text { laboratorios/talleres, } 9 \text { cubículos para } \\
\text { maestros y estación de trabajo. Centro de CAD- } \\
\text { CAM. } 2 \text { plantas. Con AC central, compresor de } \\
\text { aire seco. }\end{array}$ & $\begin{array}{l}2,275 \cdot 47 \mathrm{~m}^{2} \\
\text { Capacidad } \\
\text { para } 280 \\
\text { alumnos. }\end{array}$ \\
\hline $\begin{array}{l}\text { Auditorio y Salas } \\
\text { Audiovisuales }\end{array}$ & $\begin{array}{l}\text { Auditorio de } 280 \text { usuarios con } 6 \text { salas de } \\
\text { audiovisuales, } 4 \text { para } 35 \text { y } 2 \text { para } 70 \text { personas. }\end{array}$ & $\begin{array}{l}1,519.72 \mathrm{~m}^{2} \\
\text { Capacidad } \\
\text { para } 560 \\
\text { usuarios. }\end{array}$ \\
\hline $\begin{array}{l}\text { Centro de } \\
\text { Negocios }\end{array}$ & $\begin{array}{l}2 \text { laboratorios } 5 \text { aulas, } 26 \text { cubículos, } 7 \text { estaciones } \\
\text { de trabajo, } 2 \text { salas de juntas, cámara Gesell. } 2 \\
\text { plantas. }\end{array}$ & $\begin{array}{l}2,347 \cdot 90 \mathrm{~m}^{2} \\
\text { Capacidad } \\
\text { para } 400 \\
\text { usuarios. }\end{array}$ \\
\hline Áreas Deportivas & Áreas abiertas: 2 canchas de fútbol soccer, 1 & $17,852.39$ \\
\hline
\end{tabular}




\begin{tabular}{|l|l|l|}
\hline Edificio & \multicolumn{1}{|c|}{ Descripción } & \multicolumn{1}{|c|}{$\begin{array}{l}\text { Superficie/ } \\
\text { Capacidad }\end{array}$} \\
\hline & $\begin{array}{l}\text { cancha de fútbol rápido, 3 canchas de } \\
\text { básquetbol, Pista de jogging. }\end{array}$ & $\begin{array}{l}\mathrm{m}^{2} \text { de áreas } \\
\text { abiertas y }\end{array}$ \\
& $\begin{array}{l}\text { Edificio GYM con capacidad para 1,200 } \\
\text { espectadores y 68 usuarios, con 2 canchas de } \\
\text { usos múltiples. }\end{array}$ & $\begin{array}{l}3,635.28 \mathrm{~m}^{2} \\
\text { en edificio } \\
\text { GYM }\end{array}$ \\
\hline
\end{tabular}

Fuente: Elaboración propia con datos de Suárez (2009).

La Universidad Politécnica de San Luis Potosí define en su Política de Seguridad, Higiene y Medio Ambiente, su compromiso por asegurar la integridad física de los miembros, preservar y mejorar el medio ambiente y de contribuir al desarrollo sostenible de su entorno y sociedad. Asimismo, 2 de los 16 valores declarados en la política de calidad se refieren a la Responsabilidad Social y el Compromiso.

La huella ecológica para la Universidad Politécnica de San Luis Potosí se calcula a partir de la infraestructura de construcción, consumo de agua, energía eléctrica, residuos generados, movilidad y uso de papel, las cuales, para poder ser resumidas en un indicador general, se multiplican por factores que les permite representarse en términos de emisiones de dióxido de carbono ( $\mathrm{CO} 2$ ), y finalmente por un factor que nos permite conocer las hectáreas necesarias para compensar las emisiones generadas.

Los resultados de las emisiones de dióxido de carbono (CO2) generadas y el cálculo de la huella ecológica, son los siguientes:

\section{a. Infraestructura (construcción de edificios)}

Respecto a la infraestructura se consideró la superficie de construcción de los 14 edificios que conforman la Universidad Politécnica de San Luis Potosí, lo que representa una superficie de 60,00o m2. Asimismo, se estableció una vida útil de los edificios de 50 años, considerado como un periodo aproximado en el que no requerirán acondicionamiento que modifique el valor del factor de emisión de $\mathrm{CO} 2$. La infraestructura se mide en m2, y el resultado se multiplica por 9.5 como el factor que permite convertir el valor en términos de emisiones de dióxido de carbono (Kg de $\mathrm{CO} 2 / \mathrm{m} 2 /$ año). Asimismo, para estimar la Huella ecológica en términos de hectáreas de bosque que permitan compensar las emisiones generadas, se usa el factor 5.06 (Leyva Mas, Rodríguez Rico y Martínez Nodal, 2012). La información fue facilitada por la empresa administradora de la Universidad: Acciona (Suárez, 2009). El resultado muestra que cada año, las emisiones 
de dióxido de carbono (CO2) por la construcción de edificios ascienden a 570 Ton.

\section{b. Consumo de agua}

El cálculo sobre el consumo de agua se realiza directamente de la cifra que emite el organismo operador para la medición y cobro de uso en m3 y que, para el año 2019, ascendió a 30,344. El dato se multiplica por el factor de emisión de dióxido de carbono ( $\mathrm{CO} 2$ ), en este caso 0.50, para obtener $\mathrm{KgCO} 2 / \mathrm{Ha}$ (López Álvarez, 2009). Es importante mencionar que la Universidad Politécnica de San Luis Potosí, opera con estándares internacionales y cuenta con planta tratadora de agua, la cual recicla el $100 \%$ del agua utilizada. La información fue facilitada por la empresa administradora de la Universidad, Acciona. El consumo de agua en la universidad implica la emisión de 15.17 toneladas de $\mathrm{CO} 2$ en un año.

\section{c. Consumo de energía eléctrica}

El cálculo de la energía eléctrica se registra de las facturas generadas por la empresa proveedora en un año, y se mide en MWh, cifra que es multiplicada por el factor de emisión de dióxido de carbono (CO2), que es de 0.5, y muestra que se genera un total de 1,333.93 Toneladas de CO2/año. Asimismo, para calcular la huella ecológica se utiliza el factor 6.27 (VCS, 2014) para obtener un total de 212.75 hectáreas de bosque necesarias para compensar las emisiones generadas. La información fue facilitada por la empresa administradora de la Universidad, Acciona.

\section{d. Residuos generados}

Los residuos generados se refieren a los residuos sólidos urbanos generados durante un año (2019), y se mide en kilogramos. La información se obtuvo directamente de los registros del organismo administrador de la Universidad Politécnica de San Luis Potosí, los cuales ascienden a 34,520. La cifra se multiplica por el factor 0.61 (Leiva, 2012), para tener emisiones en términos de dióxido de carbono (CO2). La información fue facilitada por la empresa administradora de la Universidad, Acciona. El resultado muestra que para compensar los residuos generados se requiere de 3.36 hectáreas de bosque. 


\section{e. Movilidad}

El cálculo relacionado a la movilidad y uso de transporte para alumnos se realiza en kilómetros recorridos, y se distingue entre docentes y personal administrativo. Es importante mencionar que, en la Universidad Politécnica de San Luis Potosí, esta estimación se facilitó pues se dispuso del registro actualizado de los alumnos y personal docente-administrativo que disponen de vehículo, así como de su número telefónico y correo electrónico. La información faltante se obtuvo a través de una encuesta por correo electrónico y fue contestada por el $28.7 \%$ de los alumnos y por el 39.3\% del personal docente-administrativo. Se solicitó la distancia promedio que registran entre su casa y la universidad en un día hábil, resultando $4.23 \mathrm{~km}$. para alumnos y $5.75 \mathrm{~km}$. para docentesadministrativos. También se registró los que usan automóvil (25.8\% para alumnos y $91.0 \%$ en docentes-administrativos) y los que usan transporte público (57.9\% para alumnos y $6 \%$ en docentes-administrativos). Para el caso de asistencia, a los estudiantes se consideraron 190 días, tal como lo marcó el calendario escolar en el ciclo escolar 2019-2020, y para los profesores y personal administrativo se consideraron 240 días, descontando las vacaciones correspondientes al año. Finalmente, se consideró el factor de asistencia promedio, el cual para los estudiantes fue de $86.4 \%$ y para profesores y personal docente 94.2\%. Para convertir los kilómetros en emisiones en términos de dióxido de carbono $(\mathrm{CO} 2)$ se multiplicó por el factor de emisión, 0.240 y 0.070 (VCS, 2014) para uso de vehículo y de autobús, respectivamente. Los resultados muestran que la movilidad de y hacia la universidad, genera un total de 480.7 toneladas de $\mathrm{CO} 2$, lo que demanda 95 hectáreas de bosques para compensar la contaminación.

\section{f. Consumo de papel}

El cálculo de consumo de papel por parte de los alumnos se realiza en kilogramos y se obtiene a partir de encuestas donde se cuestiona sobre los montos de consumo de papel nuevo y el porcentaje de reciclaje que realizan. El cálculo de papel para personal administrativo y docente se obtiene de los registros administrativos de la Universidad y se consolida de manera anual. Los coeficientes para convertir los datos en toneladas de dióxido de carbono son 1.84, para papel nuevo y 0.65, para papel reciclado (López Álvarez, 2009). Los resultados (Tabla 9), muestran que se utiliza un total de 118 toneladas de papel nuevo y se recicla casi el 50\% del mismo, lo que provoca un total de 254.4 toneladas de dióxido de carbono (CO2), y para compensarlo se requiere de 40.6 hectáreas de bosque. 
Tabla 9. Información para el cálculo de huella ecológica de la Universidad Politécnica de San Luis Potosí

\begin{tabular}{|c|c|c|c|c|c|}
\hline Componente & Cantidad & $\begin{array}{c}\text { Emisiones } \\
\text { de CO2 } \\
\text { (Ton./año) }\end{array}$ & Factor & $\begin{array}{c}\text { Superficie } \\
\text { de bosque } \\
\text { (Ha) }\end{array}$ & $\%$ \\
\hline $\begin{array}{c}\text { Construcción de } \\
\text { edificios (m2) }\end{array}$ & $60,000.00$ & 570.00 & 5.06 & 112.65 & $24.1 \%$ \\
\hline $\begin{array}{c}\text { Consumo de } \\
\text { agua (m3) }\end{array}$ & $30,344.00$ & 15.17 & 0.50 & 2.42 & $0.5 \%$ \\
\hline $\begin{array}{c}\text { Energía eléctrica } \\
\text { (KWh) }\end{array}$ & $2,340,230.00$ & $1,333.93$ & 0.57 & 212.75 & $45.6 \%$ \\
\hline $\begin{array}{c}\text { Residuos } \\
\text { generados (Kg) }\end{array}$ & $34,520.00$ & 21.06 & 0.61 & 3.36 & $0.7 \%$ \\
\hline Movilidad (Km) & $3,701,212.83$ & 480.77 & 5.06 & 95.01 & $20.4 \%$ \\
\hline Papel bond (Kg) & $118,955.00$ & 218.88 & 1.84 & 34.91 & $7.5 \%$ \\
\hline $\begin{array}{c}\text { Papel reciclado } \\
\text { (Kg) }\end{array}$ & $58,287.95$ & 35.56 & 0.61 & 5.67 & $1.2 \%$ \\
\hline Total & & $2,675.36$ & & 466.77 & $100 \%$ \\
\hline
\end{tabular}

Fuente: Elaboración propia.

\section{Conclusiones}

Los resultados muestran que, producto de la prestación de servicios de educación superior y actividades de investigación, la Universidad Politécnica de San Luis Potosí genera un total de 2, 675.4 toneladas de dióxido de carbono, por lo que, para compensar esta contaminación, la huella ecológica le demanda una superficie de 466.77 hectáreas de bosque. Asimismo, considerando que existe una población universitaria de 5,300 personas, cada miembro debería sostener una superficie de 0.088 hectáreas de bosque ( 880 metros).

Por otra parte, considerando las fuentes de emisión de dióxido de carbono de la Universidad Politécnica de San Luis Potosí, las que mayor proporción generan son el consumo de energía eléctrica (45.6\%), las emisiones provocadas por la construcción de los edificios (24.1\%), la intensa movilidad por parte de alumnos, docentes y personal administrativo hogares-instalaciones-hogares (20.4\%), y el consumo de papel (8.7\%).

En este sentido, las actividades de responsabilidad social relacionadas con los ahorros en el consumo de la energía eléctrica, la eficiencia y optimización en los traslados hacia la universidad y la reducción en el consumo de papel, resultan oportunas al buscar incidir en el $73.4 \%$ de las fuentes de emisiones contaminantes. De manera que, suponiendo una 
política de reducción en el consumo de la energía eléctrica, la cual es el factor que aporta el 45.6\% de las emisiones de dióxido de carbono (CO2), que incluya, por ejemplo, una sustitución progresiva del uso de energía eléctrica por solar y logrando una reducción del $10 \%$, se reflejaría en una caída de $4.99 \%$ en las emisiones de $\mathrm{CO} 2$, y una reducción de $4.54 \%$ de la huella ecológica de la Universidad Politécnica de San Luis Potosí.

Finalmente, es importante reflexionar sobre la relevancia de la responsabilidad social que, a pesar de ser una práctica voluntaria, es imprescindible evitar que se convierta en una tendencia más del "Green washing”, donde las empresas "maquillan" las malas prácticas al generar un producto o servicio con un tono de mejora ambiental, aun cuando no lo sean (Correa, Flynn y Amit, 2004). En consecuencia, es fundamental incorporar mecanismos de control y verificación de los resultados de las políticas en el medio ambiente, buscando también que se formalice en el sistema de gestión y sean extensivas en grandes, medianas y pequeñas empresas a lo largo de las cadenas de valor.

\section{Referencias Bibliográficas}

Almeida Pástor, M. \& Arrechavaleta Guarton, C. N. (2018). "Responsabilidad social empresarial y sus limitaciones en el contexto académico universitario". Revista Cubana Educación Superior, 37(2), 87-103.

Altagracia Martínez, Y. (2014). La Responsabilidad Social Empresarial (RSE). España: Escuela de Organización Industrial. https://www.eoi.es/blogs/mintecon/2014/04/07/laresponsabilidad-social-empresarial-rse/ Último acceso: enero 2020.

Anand, A. (2019). "The Case for Academic Social Responsibility". Annals of Neurosciences, 26(3-4), 6-7.

Añez Hernández, C. \& Bonomie, M. E. (2010). "Responsabilidad Social Empresarial: Estrategia de Competitividad en el marco de la globalización”. Revista Formación Gerencial, 9 (1), 144-168.

Barrio Fraile, E. (2016). La Gestión de la responsabilidad social corporativa. El caso Unilever España (Tesis Doctoral). Universidad Autónoma de Barcelona, España.

Barroso Tanoira, F. (2008). "La responsabilidad social empresarial. Un estudio en cuarenta empresas de la ciudad de Mérida, Yucatán”. Revista Contaduría y Administración, 226, 73-91.

Berners-Lee, M. (2011). How Bad Are Bananas? The Carbon Footprint of Everything. Vancouver: D\&M Publishers Inc.

Cajiga Calderón, J. F. (2020). El concepto de Responsabilidad Social Empresarial. México: Centro Mexicano para la Filantropía. 
Coordinación General de Universidades Tecnológicas y Politécnicas (CGUTyP) (2020). "Cobertura de las Universidades Tecnológicas y Politécnicas por estado" https://cgutyp.sep.gob.mx/index.php?pagina=Mapa Último acceso: junio 2020.

Correa, M. E., Flynn, S. \& Amit, A. (2004). "Responsabilidad social corporativa en América Latina: una visión empresarial”. CEPAL SERIE Medio ambiente y desarrollo, 85, 16-22.

EAE Business School (2015). La responsabilidad ambiental empresarial: un asunto crítico de la RSE. España, EAE Business School. https://retos-operaciones-logistica.eae.es/la-responsabilidadambiental-empresarial-un-aspecto-clave-de-la-rse/ Último acceso: febrero 2020.

Escuela de Organización Industrial (EOI) (2012). "Ecoinnovación en procesos industriales". Wiki Escuela de Organización Industrial. https://www.eoi.es/wiki/index.php/Ecoinnovaci\%C3\%B3n_en_pro cesos_industriales Último acceso: diciembre 2019.

Etzkowitz H., Ranga, M., Benner, M., Guarany, L., Maculan, A. M. \& Kneller, R. (2008). "Pathways to the entrepreneurial University: towards a global convergence". Science and Public Policy, 35(9), 681-695.

Friedrich-Ebert-Stiftung (FES) (2019). Esto no da para más. Hacia la transformación social-ecológica en América Latina. FriedrichEbert-Stiftung. Proyecto Regional Transformación Social-Ecológica.

García Molina, S. (2019). "Propuesta de Plan de Responsabilidad Social Universitaria”. En León Borges, J. A. et al. (2019). Responsabilidad Social y Competitividad. Veracruz: Red Iberoamericana de Academias de Investigación A.C.

Ibarra Cisneros, J. M. \& Arcadio Monroy Ata (2014). "Cuestionario para calcular la Huella Ecológica de estudiantes universitarios mexicanos y su aplicación en el Campus Zaragoza de la Universidad Nacional”. TIP. Revista Especializada en Ciencias Químico-Biológicas, 17(2), 147-154.

Jiménez, I. (2014). 36 empresas con un alto compromiso ambiental. México: Forbes. https://www.forbes.com.mx/las-36-empresas-con-un-altocompromiso-ambiental/ Último acceso: noviembre 2019.

León Borges, J. A., Makita Balcorta, T. G., Gaber Bustillos, V. A., Flores Castro, E. C., García Díaz, L. de J., Góngora Avalos, G. del C. \& Puente Guzmán, G. (2019). Veracruz: Responsabilidad Social y Competitividad. Red Iberoamericana de Academias de Investigación A.C.

Leiva Mas, J., Rodríguez Rico, I. L. \& Martínez Nodal, P. (2012). "Cálculo de la huella ecológica en universidades cubanas. Caso de estudio: Universidad Central "Marta Abreu" de Las Villas", AFINIDAD LXVIII, 557, 30-34. 
López Álvarez, N. (2009). Metodología para el Cálculo de la huella ecológica en universidades. Congreso Nacional del Medio Ambiente. Universidad de Santiago de Compostela. Oficina de Desarrollo Sostenible.

Medina, L. M. (2006). "La responsabilidad social de la empresa”, Ponencia presentada en el $X$ Congreso Anual de la Academia de Ciencias Administrativas A.C. (ACACIA). Universidad Autónoma de San Luis Potosí. San Luis Potosí.

Ministerio del Medio Ambiente (MMA) (2020). Huella de carbono. Santiago, Chile: Ministerio del Medio Ambiente. https://mma.gob.cl/cambio-climatico/cc-02-7-huella-de-carbono/ Último acceso: enero 2020.

Martínez Castillo, R. (2008). "Educación y huella ecológica". Revista Electrónica "Actualidades Investigativas en Educación", 8(1), 1-28.

Martínez Ruiz, M. A. (2013). Indicadores como información base para el análisis del Desempeño ambiental: huella hídrica, huella ecológica y Huella de carbono (Tesis de Maestría publicada). Universidad Autónoma de Nuevo León, México.

National Aeronautics and Space Administration (NASA) (2020). NASA Selects New Instrument to Continue Key Climate Record. Washington D.C., United States: NASA. https://www.nasa.gov/press-release/nasa-selects-new-instrumentto-continue-key-climate-record Último acceso: marzo 2020.

ONU-Habitat (2016). Urbanización y Desarrollo. Futuros Emergentes. Reporte de Ciudades del Mundo, 2016. Programa de las Naciones Unidas para los Asentamientos Humanos.

Observatorio de Responsabilidad Social Corporativa (ORSC) (2014). Introducción a la Responsabilidad Social Corporativa. Observatorio de Responsabilidad Social Corporativa.

Pérez Chavarría, M (2009). "Responsabilidad social corporativa (RSC) y comunicación: la agenda de las grandes empresas mexicanas". Signo y Pensamiento, XXVIII(55), 201-127.

Ruiz Barrios, J. L. (2013). Diseño de modelo de Responsabilidad Social Empresarial en Pyme constructora Araucana. (Tesis de Maestría publicada). Universidad Nacional de Colombia, Colombia.

Suárez, S. (2009). Acciona concesiones: Proyecto de provisión de servicios (PPS) Universidad Politécnica de San Luis Potosí. https://publications.iadb.org/es/publicacion/15010/accionaconcesiones-proyecto-de-provision-de-servicios-pps-universidad Último acceso: septiembre 2020.

United Nations (UN) (2015). Agenda 2030 para el Desarrollo Sostenible. Organización de las Naciones Unidas.

Vicerrectorado de Campus y Sostenibilidad (VCS) (2014). Huella Ecológica de la Universidad de Málaga. Vicerrectorado de Campus y Sostenibilidad. 
Wackernagel, M \& Rees W. (1996). Nuestra huella ecológica: reduciendo el impacto humano sobre la Tierra. Santiago de Chile: Ediciones LOM.

World Wildlife Fund (WWF) (2018). Informe planeta vivo 2018, Panorama global. Gland, Suiza: World Wildlife Fund (WWF). http://www.wwf.org.mx/quienes_somos/planeta_vivo/ Último acceso: julio 2020.

\footnotetext{
${ }^{1}$ Se refiere a pequeñas empresas o talleres usualmente informales que no cumplen con la regulación legal, intensivas en mano de obra, donde los empleados reciben bajos niveles salariales, sin seguridad social, con largas jornadas de trabajo, en malas y riesgosas condiciones laborales. Pueden llegar a ser parte de una cadena de proveeduría como en la industria de la confección u otras empresas internacionales.

2 Para consumo de agua, construcción de edificios, energía eléctrica, consumo de papel y generación de residuos y movilidad.

3 Se consideran hectáreas de bosque considerando que la fotosíntesis realizada por la vegetación representa la principal forma de captura del dióxido de carbono, por lo que se considera como pago por servicios ambientales.

4 Se refiere a aquellas personas o grupos de personas que están directa o indirectamente vinculados con la operación de la empresa, pudiendo ser desde el conjunto de empleados, proveedores primarios o secundarios, clientes, proveedores de servicios especializados, inversionistas e incluso áreas de gobierno, organismos empresariales, ONG o aquellos participantes en la cadena de valor de la empresa.

5 A partir de 2002 se desarrolló en México el esquema denominado "Proyecto para la Prestación de Servicios" (PPS), que consiste en un acto jurídico entre una entidad o dependencia de la Administración Pública Federal y una empresa privada, en el que se involucran recursos, riesgos y recompensas, para que esta última preste servicios a largo plazo con activos que construya con sus recursos sobre terrenos propios o de un tercero, y por los cuales la entidad o dependencia realizará pagos a largo plazo, conforme al contrato del PPS (Auditoria Superior de la Federación, 2015).

${ }^{6}$ Gestiona en apego a los 10 principios de Ecuador.

7 La fecha de inicio de la construcción de la Universidad Politécnica de San Luis Potosí, primera etapa, fue el martes 24 de julio de 2007 y la fecha de término el día miércoles 23 de marzo de 2011.
} 